\title{
Development of High-Throughput, High-Resolution 3D Reconstruction of Large- Volume Biological Tissue Using Automated Tape Collection Ultramicrotomy and Scanning Electron Microscopy
}

\author{
R. Schalek*, N. Kasthuri**, K. Hayworth**, D. Berger***, JC Tapia**, JL. Morgan**, S.C.

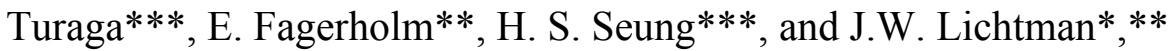 \\ * Center for Brain Science, Harvard University, 52 Oxford St., Cambridge, MA 02138 \\ ** Department of Molecular and Cellular Biology, Harvard University, Cambridge, MA 02138 \\ *** Department of Brain and Cognitive Science and Physics, Massachusetts Institute of Technology, \\ Cambridge, MA 02139
}

A full understanding of brain function requires extensive knowledge of the intricate patterns of axons and dendrites that connect neurons at synapses. Such wiring diagrams ("connectomes") are in short supply owing to the enormous number of synaptic connectivities that need to be catalogued and the very high resolution necessary to trace them [1]. The key therefore is to have an approach that both allows large volumes (cubic millimeters or larger) to be analyzed but at a level of resolution of several nanometers. One approach to this problem is to slice the brain into many thousands of very thin sections and then reconstruct the connectome by tracing nerve cell processes (axons and dendrites) from one section to the next. Obviously the sheer number of sections, digital images and the millions or more processes that need to be traced requires an automated approach. We have approached this problem by automating a number of steps with the ultimate aim of having a fully automated pipeline from tissue sample to wiring diagram.

The suite of techniques we are developing have the long-term goal of allowing us to eventually tackle the connectome of an entire animal such as a mouse brain (vol $\sim 1 \mathrm{~cm}^{3}$ ). Three different aspects of the pipeline are being worked on: (1) sample preparation, sectioning and collection, (2) imaging and image data management, and lastly (3) image segmentation . Our approach utilizes slightly modified traditional EM techniques of sample preparation and sectioning, but uses a conveyor belt device to automatically collect the sections on a continuous tape. The unattended automated tape ultramicrotome (ATUM) sectioning creates a lossless collection of sections on a flexible substrate that are then automatically imaged using either backscattered electrons or secondary electrons in a FESEM. The basic steps are depicted in Figure 1.

In Figure 2 we present results obtained from 2100 nondestructively imaged, $29 \mathrm{~nm}$ thick sections of enbloc stained adult mouse cortex. To obtain the larger context of a small area imaged at high resolution, four multiscale resolution data sets from the same sample were collected: (1) $48 \mu \mathrm{m} \times 48$ $\mu \mathrm{m}$ FOV, $3 \mathrm{~nm}$ per pixel 16k x 16k images, $2 \mu \mathrm{s}$ dwell time; (2) $300 \mu \mathrm{m} \times 300 \mu \mathrm{m}$ FOV, $30 \mathrm{~nm}$ per pixel 4k x 4k images, $1 \mu \mathrm{s}$ dwell time; (3) $1000 \mu \mathrm{m}$ x $600 \mu \mathrm{m}$ FOV, $30 \mathrm{~nm}$ per pixel 4k x 4k images taken every eighth section, $1 \mu \mathrm{s}$ dwell time; and (4) $1.5 \mathrm{~mm} \times 2.5 \mathrm{~mm} \mathrm{FOV}, 750 \mathrm{~nm}$ per pixel overview images. A combination of human segmentation and automated machine learning segmentation has been performed on a subset of the data sets. In addition to imaging and analysis of the cortical dataset, other biological systems such as C-elegans, bacteria, and olfactory bulb have been imaged. To speed the imaging we have examined secondary electron imaging and found that with appropriate sample preparation produces high quality images with dwell times of $25 \mathrm{~ns}$. At these imaging speeds the time to reconstruct an entire mouse brain would require many years and 
many microscopes operating in parallel. Increasing imaging speed using a multibeam SEM (RAPIT prototype, Zeiss) should substantially decrease the required imaging time. The authors would like to thank the sponsors of the research [2].

\section{References}

[1] N. Kasthuri and JW Lichtman, Neuropsychopharmacology, 35(1) (2010) 324.

[2] This research was supported by grants from the Gatsby Charitable Trust, a Howard Hughes Collaborative Innovation Award (X. Zhuang PI) and the NIH.

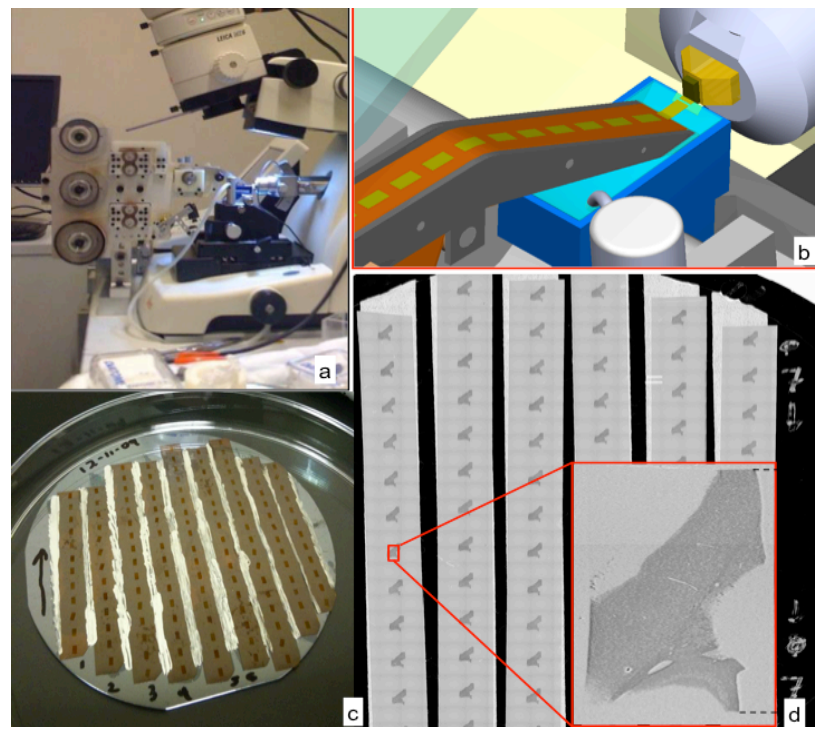

Figure 1: (a) Automated tape ultramicrotome (ATUM), (b) CAD drawing of the section collection process, (c) sections on polyimide tape mounted onto a silicon wafer, (d) BSD mosaic image of the sections on the wafer (inset shows a higher magnification of an individual section).
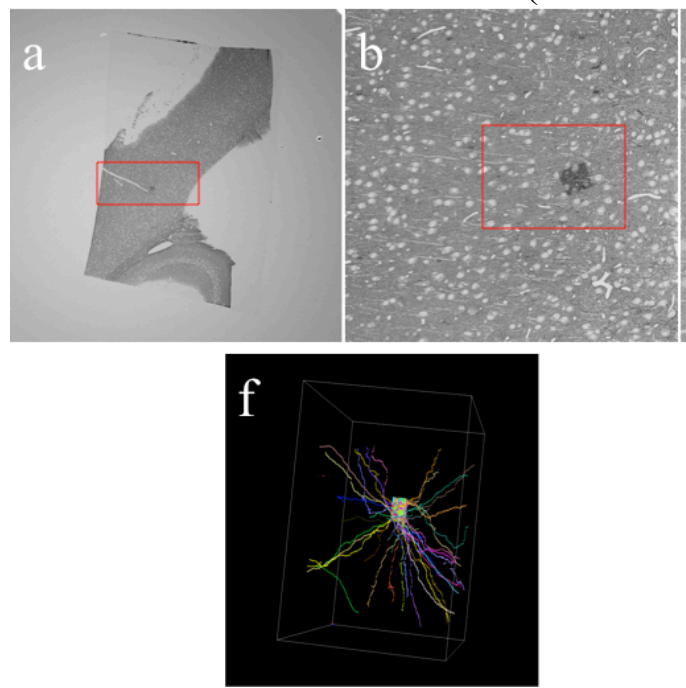
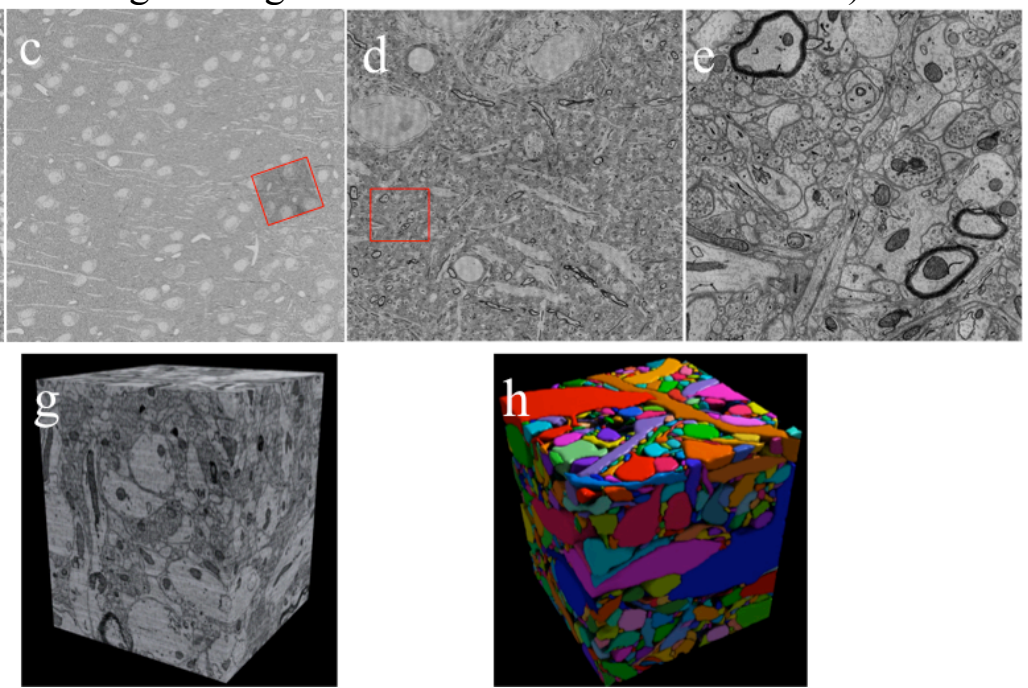

Figure 2: (a) $\sim 1.5 \mathrm{~mm} \times 3 \mathrm{~mm}$ FOV overview image, (b) 1 $\mathrm{mm}$ x $0.6 \mathrm{~mm} \mathrm{FOV,} \mathrm{(c)} \sim 300 \mu \mathrm{m} \times 300$ $\mu \mathrm{m}$ FOV, (d) $\sim 8 \mu \mathrm{m}$ x $48 \mu \mathrm{m}$ FOV, (e) $\sim 4 \mu \mathrm{m}$ x $4 \mu \mathrm{m}$ FOV, (f) partial segmentation of $\sim 300 \mu \mathrm{m} \times$ $300 \mu \mathrm{m} \times 60 \mu \mathrm{m}$ reconstruction, (g) $5 \mu \mathrm{m}$ x $5 \mu \mathrm{m}$ x $5 \mu \mathrm{m}$ reconstruction, and (h) fully segmented block of tissue shown in $(\mathrm{g})$. 